\title{
A Corrected EMF Problem
}

\author{
Frederick J. Young \\ Division of Communications and Arts, University of Pittsburgh, Bradford, PA, USA \\ Email: youngfj@youngbros.com
}

How to cite this paper: Young, F.J. (2018) A Corrected EMF Problem. Open Access Library Journal, 5: e4270.

https://doi.org/10.4236/oalib.1104270

Received: December 19, 2017

Accepted: January 28, 2018

Published: January 31, 2018

Copyright $\odot 2018$ by author and Open Access Library Inc.

This work is licensed under the Creative Commons Attribution International License (CC BY 4.0).

http://creativecommons.org/licenses/by/4.0/

\begin{abstract}
An error in The Lectures on Physics by Feyman, Leighton and Sands is discussed and corrected. It is caused by the improper application of Faraday's induced voltage law.
\end{abstract}

\section{Subject Areas}

Applied Physics

\section{Keywords}

Feynman EMF Mistake, EMF, Faraday's Law, Moving Conductor

\section{Introduction}

There exists a great amount of confusion about the calculation of electromotive force commonly denoted as EMF. The introductory treatment for the calculation of EMF as presented in most elementary texts is inadequate both because the quantities often are not carefully defined and because the restrictions upon the validity of the formulas given are not emphasized. A fallacious understanding of Faraday's law is given in many texts and in various internet sources. This short paper considers the EMF generated by plates rocking in a uniform magnetic field. This problem is taken from Feynman et al. [1]. This text erroneously calculates the EMF and cannot verify it experimentally. Many other texts also contain erroneous EMF calculations without any attempt to verify.

\section{Emf Generated by Plates Rocking in a Uniform Magnetic Field [2]}

In Figure 1 two copper plates with slightly curved edges are rocking back and forth between the solid and the dashed lines in a uniform magnetic induction field directed into the paper. Each plate is connected to one of the terminals of a voltmeter or galvanometer. The plates make contact at the point P initially 


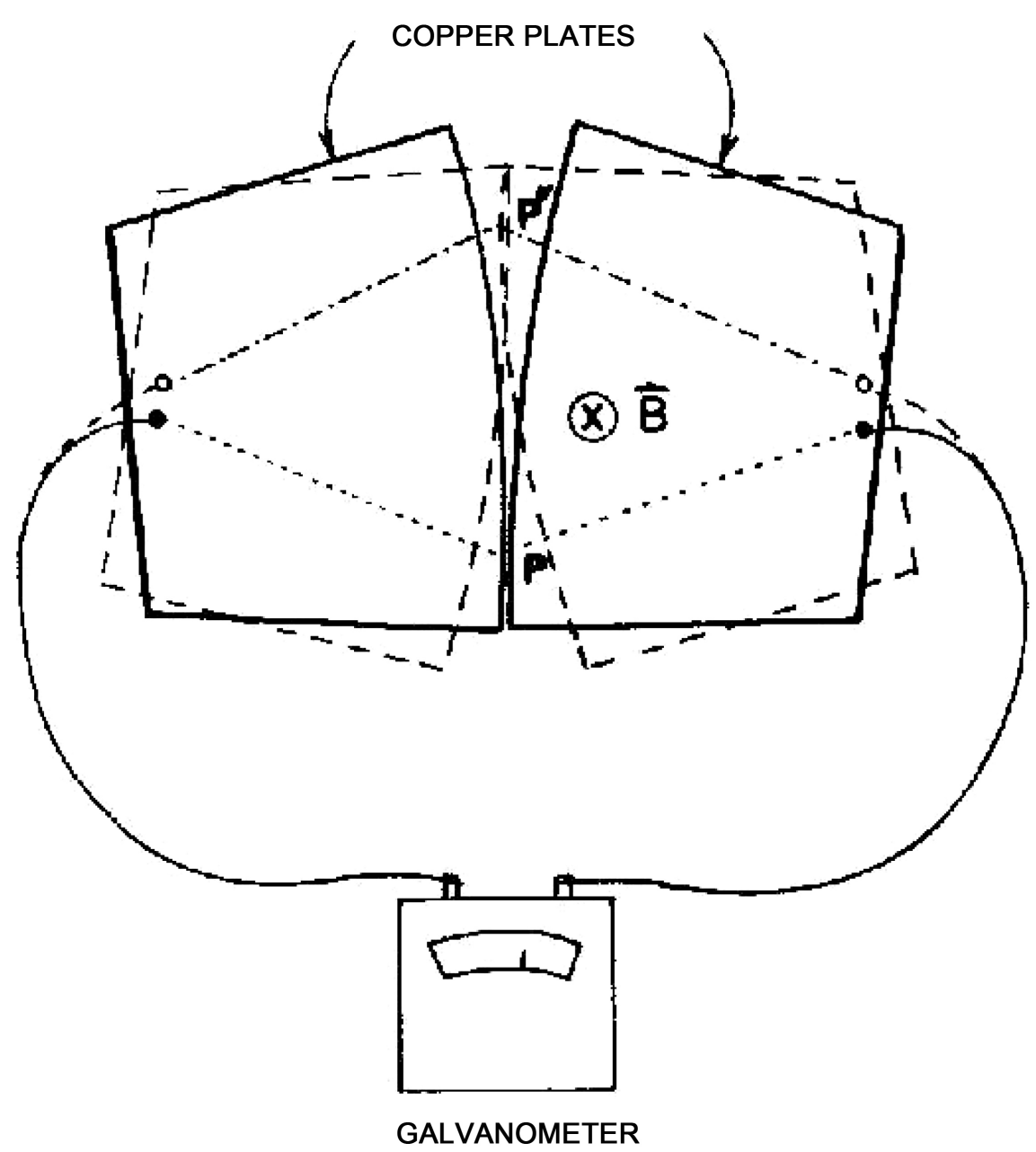

Figure 1. Rocking plates.

making a complete circuit including the galvanometer. As the plates are rocked the place of contact moves continuously from point $P$ to point $P^{\prime}$. If we imagine the circuit to be completed through the plates on the dotted line shown in the figure, the magnetic flux through this circuit changes by a large amount as the plates are rocked. Feynman, Leighton and Sands [1] given said large flux change, wrongly claim this is an exception to Faraday's Law and a measurable EMF should be induced. They measure no EMF and show a complete lack of physical understanding of the calculation of EMF by Faraday's law. Here they indicate that the circuit in the plates moves between the dotted and dash-dot lines and makes a $\boldsymbol{V} \times \boldsymbol{B}$ that can be minimized by making $\boldsymbol{V}$ very small. However, they say there may still be a large change in flux linking the circuit. The simplest way to solve for the EMF is to place the observers on both plates. They will each say their plate is stationary and not moving in a magnetic field. However, they will notice the leads and galvanometer moving with respect to them. The observer on the left plate will see the right plate moving twice as fast as an observer on the galvanometer and he would see a small $2 \boldsymbol{v} \times \boldsymbol{B}$ in the connection made to the right plate and none in his connection. He would also see a small change 
in flux in the area between the dashed and dotted lines comprising the leads on the right side. This would lead to the very small potential Feynman et al. observed. If the leads were stiff and the contacts were connected to brushes at the edge of each plate the leads would not move and there would be no EMF at all generated. A slightly different configuration of these plates is shown in Figure 2. It has no leads that move as the plates are rocked. The line abcdefgh shows the base of the red plates at rest and that line is the most direct way for current to flow to the red brushes and around the loop through the voltmeter. There is a magnetic induction, $\mathbf{B}$ directed into the paper. The plates are moved approximately fifteen degrees and are depicted in that position by dashed lines and the part that would stick out where there are two sets of plates is colored a light green. Initially the path from the voltmeter is given by abcdefgh. As the plates move from the initial position the path becomes abcije'klgh around the loop through the voltmeter. Along this path $-\mathrm{d} \Phi / \mathrm{d} t=0$. The only point at which the right and left plates touch is at point e'. Faraday's Law can be applied only if there are no breaks in the path. The $\boldsymbol{v} \times \boldsymbol{B} \cdot \mathrm{d} l$ on the left plate cancels the same term on the right plate. Thus Faraday's law yields zero EMF. An observer might be stationed on either the left or the right conducting plate or on the external circuit comprising the brushes and the voltmeter leads. As illustrated many times [2] [3], all observers must agree on the EMF produced. The ones sitting on the plates would have to completely evaluate

$$
\mathrm{EMF}=-\int_{S} \partial \boldsymbol{B} / \partial \boldsymbol{t} \cdot \mathrm{d} \boldsymbol{S}+\oint \boldsymbol{v} \times \boldsymbol{B} \cdot \mathrm{d} \ell
$$

along a path and embraced area including the brushes and voltmeter leads. This equation is derived by Young [3]. The derivation is repeated below. The observer on the external circuit, however, sees no time rate of change in the magnetic induction flux if he takes the correct path. His circuit goes counter clockwise from the left terminal of the voltmeter to the left brush, passing through points abcdeflgh through the right brush to the right terminal of the voltmeter. Thus the $\mathrm{EMF}=0$ regardless of the movement of the plates and independent of their magnetic relative permeability. This is the easy solution.

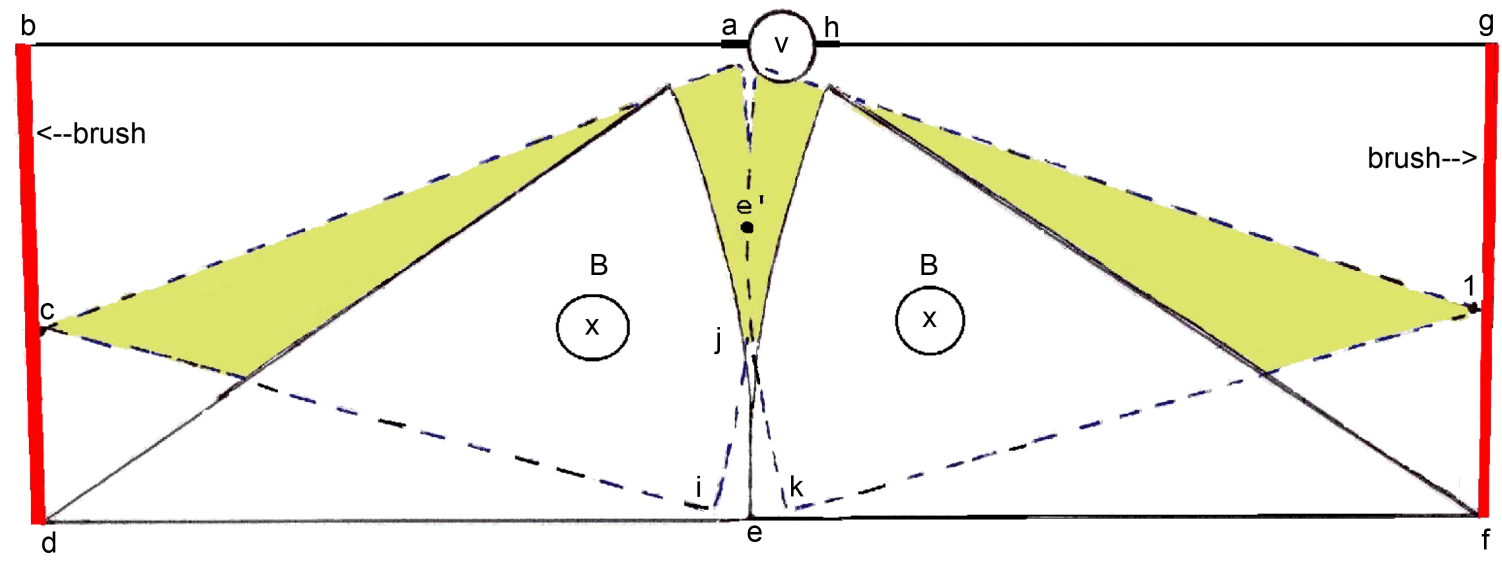

Figure 2. Rocking plates with brushes. 


\section{Electromotive Force with Relative Motion between Circuit Parts}

We develop a definition for the electromotive force about any closed metallic circuit whose various parts may be moving relative to each other and which may or may not contain sliding contacts. Once again the desired definition of EMF must be consistent with Kirchhoff's Law. When there is relative motion between parts of the circuit the resistance of a segment must be defined in such a way that it does not change if the segment is moving. That is, if one wishes to retain the formula for the dissipation in an element as $I^{2} R$ and because both the dissipation and the current are independent of the observer, it follows that the resistance to be used in this formula should be the same as the resistance calculated by an observer stationary with respect to that element. In that case the resistance of any element is defined as

$$
R_{i}=\left(\int_{i} \boldsymbol{E}^{\prime} \cdot \mathrm{d} l\right) /\left(\int_{i} \boldsymbol{J}^{\prime} \cdot \mathrm{d} \boldsymbol{S}\right)
$$

where the primes denote that the fields are measured by an observer at rest with respect to the conductor at the point $\mathrm{d} l$. (if the conductor is flexible it can be considered as comprising an infinite number of increments each of which has a resistance that is evaluated by an observer on that particular increment.) Because the current flowing through a conductor is independent of the observer, the $I R$ drops about the circuit can be written as

$$
\begin{aligned}
\sum_{i} I_{i} R_{i} & =\left\{\sum_{i}\left(\int_{i} \boldsymbol{J}^{\prime} \cdot \mathrm{d} \boldsymbol{S}\right) /\left[\left(\int_{i} \boldsymbol{E}^{\prime} \cdot \mathrm{d} l\right) /\left(\int_{i} \boldsymbol{J}^{\prime} \cdot \mathrm{d} \boldsymbol{S}\right)\right]\right\} \\
& =\sum_{i} \int_{i} \boldsymbol{E}^{\prime} \cdot \mathrm{d} l=\oint \boldsymbol{E}^{\prime} \cdot \mathrm{d} l
\end{aligned}
$$

It thus appears that a reasonable definition of the electromotive force about any prescribed path in a non-rigid, conducting circuit is given by

$$
e=\oint \boldsymbol{E}^{\prime} \cdot \mathrm{d} l
$$

where $\boldsymbol{E}^{\prime}$ is defined as the electric field intensity evaluated at the element $\mathrm{d} l$ by an observer stationary with respect to the conductor at that point. To avoid having to use several reference frames in evaluating Equation (4),

$$
\boldsymbol{E}^{\prime}=\boldsymbol{E}+\boldsymbol{v} \times \boldsymbol{B}
$$

Equation (5) from the special theory of relativity can be used to transform each element of Equation (4) to single given reference system. The fields measured by an observer in this fixed reference frame will be denoted by the unprimed quantities. By solving Equation (5) for $\boldsymbol{E}$ and substituting it into the Maxwell equation $\nabla \times \boldsymbol{E}=-\partial \boldsymbol{B} / \partial \boldsymbol{t}$, there results

$$
\nabla \times \boldsymbol{E}^{\prime}=-\partial \boldsymbol{B} / \partial \boldsymbol{t}+\nabla \times(\boldsymbol{v} \times \boldsymbol{B})
$$

Integrating both sides of Equation (6) over the surface enclosed by the path about which the EMF is to be calculated and applying Stokes Theorem there re- 
sults

$$
e=-\int_{S} \partial \boldsymbol{B} / \partial \boldsymbol{t} \cdot \mathrm{d} \boldsymbol{S}+\oint \boldsymbol{v} \times \boldsymbol{B} \cdot \mathrm{d} l
$$

where $\boldsymbol{B}$ is the total magnetic induction field due to all causes and has been assumed to be independent of the observer.

$v$ is the velocity of the segment of the conductor at the point corresponding to $\mathrm{d} l$ relative to some given observer. (Note carefully that $v$ is the velocity of the conductor and not the velocity of the path element $\mathrm{d} l$ in case the two differ as will be shown in later examples.)

$\partial \boldsymbol{B} / \partial \boldsymbol{t}$ is the vector time rate of change of $\boldsymbol{B}$ relative to the same observer.

Warning!! This formula has been derived from the results of the Special Theory of Relativity and hence is strictly justified only for the case of uniform motion. However, it is found experimentally to hold with negligible error for all accelerations that are physically attainable in the terrestrial laboratory. The resulting error only can be found from The General Theory of Relativity.

Once again the expression for the electromotive force (EMF) as exhibited in Equation (7) contains a surface integral and hence can be evaluated only for a closed circuit. It is nevertheless possible to define a unique potential difference between the terminals of a generator, just as in the case of rigid circuits. These terminals and the external circuit must lie within a region that to some observer is irrotational and that observer must see no part of the external circuit moving through a magnetic field. In other words, if it is possible to connect these terminals with a rigid line so that there is no EMF in any closed circuit formed by this line and any permitted external circuit, then it is possible to uniquely define a terminal voltage which is equal to the EMF (computed about the internal circuit and this rigid line) minus the IR drop in the generator: e.g. $V_{A B}=-\int_{A}^{B} E \cdot \mathrm{d} l$. As before the sign convention is such that the line integral of Equation (4) is taken as positive in the direction going from the negative terminal, $A$, through the generator to the positive terminal, $B$, and $\int_{A}^{B} \boldsymbol{E}^{\prime} \cdot \mathrm{d} l^{\prime}$ is taken from $A$ to $B$ through the generator.

This section is restricted to cases where any segment of the circuit moving relative to any observer through a magnetic field is a conductor. The case of a dielectric moving through a magnetic field has been presented by Young [3].

\section{Conclusion}

It is best not to mix special relativistic equations such as Equation (5) with Faraday's law that was established more than a century earlier. That was the mistake made by Feynman et al. [1] when they thought EMF was generated along the arbitrary paths given in Figure 1 as dotted paths moved to the dash-dotted paths through the perpendicular magnetic field. They did not understand when there is conductor motion the flux variation must be bounded by the path where cur- 
rent is expected to flow. Numerous examples are given in the scientific literature [2] [3].

\section{References}

[1] Feynman, R.P., Leighton, R.B. and Sands, M. (1964) The Feynman Lectures on Physics, The EM Field. Addison-Wesley Publishing Company, Inc., Reading, Massachusetts, 17-2 - 17-3.

[2] Hughes, W.F. and Young, F.J. (1966) The Electromagnetodynamics of Fluids. John Wiley \& Sons, Inc., New York, 28-33.

[3] Young, F.J. (2012) Magnetically Induced Electromotive Force. Xlibris Corp. http://www.Xlibris.com 